\title{
Referential Redundancy and the Integration of Verbal Information
}

\author{
Melvin Manis and Marjorie B. Platt \\ University of Michigan and Ann Arbor Veterans Administration Hospital
}

Received February 10, 1975

\begin{abstract}
In two experiments. college students read pairs of messages describing an actor's portrayal of a particular emotion. They were then asked to identify the one of 24 target photographs about which each message-pair was written. When the respondents' selections were made immediately after reading the message-pair. accuracy was inversely related to the redundancy of the two passages. Performance on the nonredundant pairs deteriorated relatively rapidly, however, when an arithmetic task was interpolated between the receipt of the message-pair and the presentation of the referent array from which the target was to be selected; by contrast, the redundant pairs elicited a stable performance pattern that was essentially unaffected by the interpolated arithmetic task. In a third experiment, each subject served as a transmitter. He was shown a target-photograph together with a "given" description and was asked to "supplement" it with a second description to produce a message-pair that would enable a recipient to identify the proper target-photograph in a decoding task of the type used in Experiments I and II. Given a choice between two "additional" descriptions that produced identical hit-rates when presented singly, respondents generally selected the passage that was more redundant with the "given" description.
\end{abstract}

This paper is concerned with the role of redundancy in two important communication settings. Experiments I and II explore the effects of redundancy in a decoding task, where the respondent's goal is to identify the referent implied by a pair of descriptive messages. Experiment III treats redundancy as a dependent variable; it assesses the extent to which people choose redundant (versus nonredundant) messages when describing a referent stimulus to others.

Redundancy has frequently been invoked as an explanatory concept in analyses of language and cognition. In the studies reported below, we have been exclusively concerned with referential redundancy - the extent to which two extended passages do (or do not) imply the same referent(s). The conceptual definition of redundancy implies, other things being equal, that a redundant pair of messages will contain less total information than a

Requests for reprints should be scnt to Melvin Manis. Research Center for Group Dynamics, Institute for Social Research, University of Michigan, Ann Arbor, Michigan 48106. All statements are those of the authors and do not necessarily reflect the opinions or policy of the Veterans Administration. 


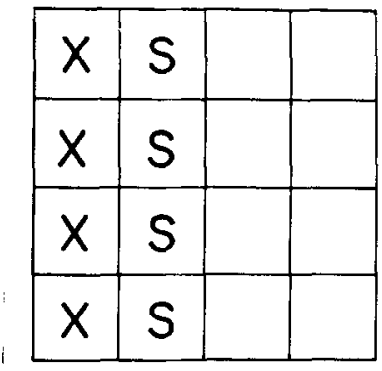

Fig. 1. A hypothetical example demonstrating the concept of referential redundancy (see text).

nonredundant pair. This leads rather directly to the prediction that nonredundant message-sets (message-pairs in the present case) should produce more accurate communication than redundant pairs. The basis for this prediction can be shown rather clearly if we consider Fig. 1.

Suppose a respondent (receiver) is asked to locate the position of an unseen target referent, $T$, in the $4 \times 4$ matrix shown in Fig. 1; assume he is provided with two messages: (a) "T is on the far left," and (b) "T is in a cell containing an $X$." Since (a) and (b) each refer (ambiguously) to the same four cells, the two messages are referentially redundant; if forced to make a choice, the average respondent would have a $25 \%$ chance of selecting the proper cell. In contrast, suppose the receiver is given the following two messages: (a) "T is on the far left," and (b) "T is in the topmost row." Considered individually, these messages are just as informative as the messages in the first pair; that is, each one is consistent with four potential referents. In this case, however, the two messages imply somewhat different sets of referents (cells), and hence they are relatively nonredundant. Since there is just one cell that is consistent with both descriptions, this nonredundant message-pair should enable an intelligent respondent to locate the intended target unerringly.

This conception of the redundancy variable is closely related to the terminology that has been adopted in studies of concept formation, where two or more attributes are regarded as redundant if they have a disproportionately high frequency of co-occurrence (Trabasso \& Bower. 1968). For example, the attributes red and square would be redundant in a concept formation experiment if all of the red stimuli were also square. Note that in this case, if a particular stimulus (referent) were described as red by one person and square by another, receipt of the second description (square) would not reduce the uncertainty of a person who had already received the first description and was trying to identify the appropriate target referent.

Manis and van Rooijen (1973) explored the relationship between referential redundancy and the respondents' ability to identify the referents 
that were described in several pairs of written descriptions. The passages in each pair were based on the same referent (a photograph of an actor in an emotional pose). The respondents' tasks were to read each message-pair and select the particular photograph that appeared to be the referent for that pair from an array that included 24 different photographs. Some message-pairs contained descriptions that were relatively redundant, in the sense that when presented individually they elicited similar response profiles (i.e., they were associated with similar alternatives); other message-pairs were relatively nonredundant. The results indicated that the redundant message-pairs yielded lower hit-rates than the nonredundant pairs. Experiments $I$ and II in the present series were designed in part to replicate the Manis-van Rooijen results.

\section{REDUNDANCY AND FORGETTING}

Experiments I and II were also designed to assess the impact of forgetting on our respondents' capacity to identify the referents for redundant versus nonredundant message-pairs. Two lines of analysis suggest that forgetting may produce a relatively mild performance decrement when redundant messages are presented for decoding.

\section{A Structural Theory}

Redundancy is believed to safeguard communication when messages are transmitted in a "noisy" environment. Under these conditions, even if a component part of a given message is forgotten (or not received due to interruption, faulty hearing, etc.), it need not impair comprehension unduly, for the redundancy of the message may enable a receiver to infer the significance of the parts that are missing. Thus, suppose two messages are redundant. Then, even though part of one message may subsequently not be recalled, decoding accuracy might be relatively unaffected, since similar choice-relevant information would be available from the description that was recalled. In contrast, if the messages are not redundant, information that is forgotten from one passage is unlikely to be represented in the other, and thus decoding accuracy may deteriorate. Let us illustrate this point more concretely with reference to the problem represented in Fig. 1.

Example 1: Partial forgetting. Assume again that a respondent receives a pair of redundant descriptions depicting the target referent as (a) on the far left and (b) in a cell with an $X$ and is asked to locate the referent in one of the cells of Fig. I. Since four cells are consistent with these descriptions, a redundant message-set of this type should yield a $25 \%$ hit-rate. However, suppose now that with the passage of time, the respondent remembers only a generalized version of the two descriptions and recalls the target as being $\left(a^{\prime}\right)$ in the left half of the figure and $\left(b^{\prime}\right)$ in a cell that includes either an $X$ or 
an $S$. In this garbled version, the information that is recalled is consistent with eight different locations, and hence we would now anticipate a $12.5 \%$ hit-rate ( 1 out of 8 ), which represents a decrement of $12.5 \%$.

Now consider a nonredundant message-pair: (a) the target is on the far left; (b) the target is in the topmost row. Consider what would happen if, through the vagaries of forgetting, our respondent remembered the target as being $\left(\mathrm{a}^{\prime}\right)$ in the left $h a l f$ and $\left(\mathrm{b}^{\prime}\right)$ in the top half of the figure. In this case, four cells fit both descriptions, and hence the hit-rate should decrease to $25 \%$ ( 1 out of 4 ), a decline of $75 \%$ from the errorless performance that would result from perfect recall. Note that this decrement is much greater than the $12.5 \%$ decrement that was anticipated from a comparable retention loss, given a redundant message-pair. Nonetheless, other things being equal, a purely structural theory suggests that so long as something is retained from each of the input passages, a nonredundant message-pair should generate better performance than a comparable pair of redundant messages $(25 \%$ versus $12.5 \%$ in the present example).

Example 2: Complete forgetting of one passage. Now assume that passage (b) in each of the message-pairs has been completely forgotten, while passage (a) has been generalized to (a'), i.e., the target is in the left half of the figure. Since $\left(a^{\prime}\right)$ is consistent with eight different cells, it should yield a hit-rate of $12.5 \%$ ( 1 out of 8 ), regardless of the information that had been encoded in (b), its now forgotten "mate." Note, however, that this purely structural analysis does not lead us to anticipate a situation where nonredundant message-pairs ultimately produce a decoding performance that is inferior to the performance elicited by redundant pairs. Instead, the theory predicts a gradual convergence of results.

\section{A Theory Based on Retention Differences}

Redundant message-pairs may be more easily remembered than nonredundant pairs. Previous studies have shown that word lists based on related elements can be recalled more accurately than comparable lists of unrelated words (Deese, 1965, p. 60; Underwood, 1964). Since the component passages in a redundant message-pair are more closely related in the information they convey than are nonredundant passages, they should be retained more effectively. As a consequence, redundant message-pairs should generate relatively stable performance patterns when they are stored in memory.

In contrast to the structural theory, the retention analysis suggests that nonredundant message-pairs might ultimately yield low'er hit-rates than the redundant pairs, although this effect is not "required" by the theory. That is, since a nonredundant pair of descriptions might be forgotten fairly rapidly, the diagnostic advantage (higher hit-rate) normally enjoyed by such a pair might decline and ultimately be reversed. Thus, to return to our previous illustration (see Fig. 1 and the associated text), it is conceivable 
that following an extended retention interval, passage (b) might be completely forgotten if it was presented as part of a nonredundant pair, while passage (a) might be remembered in the garbled form we have previously referred to as $\left(a^{\prime}\right)$. As noted above, a scenario like this would lead us to anticipate a $12.5 \%$ hit-rate. By contrast, if passages (a) and (b) were redundant and hence easy to remember, they might be recalled with essentially perfect fidelity, yielding a hit-rate of $25 \%$. In brief, despite the initially higher performance level that would be anticipated from a nonredundant message-pair, if the component passages in such a pair were forgotten at a sufficiently rapid rate, the introduction of an extended delay period might ultimately yield a "crossover" (that is, better performance on redundant than on nonredundant message pairs).

Experiments I and II are addressed to the issues described above; that is, our interest was centered on the message receiver, faced with the problem of inferring the referent implied by a pair of descriptive messages. The theoretical focus changes in Experiment III, which is concerned with redundancy as a property of the communicator's behavior. The main purpose of this experiment was to assess the redundancy of the communicator's output, when placed in a situation that required him to supplement an imperfect descriptive passage that was already a vailable to his listener.

\section{EXPERIMENTS I AND ||}

Following the rationale that is presented above, Experiments I and II were designed to explore the impact of referential redundancy on the respondents" ability to identify the appropriate referents in a series of message-pairs. Performance was assessed both immediately after receipt of the selected message-pairs and after an interpolated delay period.

\section{Method}

Experiments I and II were similar in design. A large pool of written descriptions that were derived from another study (Manis \& Armstrong. 1971) was first decoded by several norm groups to establish the distribution of referent-choices associated with each passage. To assess referential redundancy, we compared the response-profiles that the various passages elicited when presented singly, using the $D$-statistic to quantify similarity (Cronbach \& Gleser, 1953; Osgood \& Suci, 1952). When a pair of descriptions elicited similar choice-patterns, they were regarded as redundant, since these passages seemingly implied the same referents and in this sense conveyed the same information.

\section{Selection of Message-Pairs}

To assemble high- and low-redundancy message-pairs while controlling for differences in the hit-rates elicited by the component passages, the following procedure was adopted. First, for each referent photograph we identified those passages that produced the same hit-rates when presented in isolation. Then, each of two matched descriptions ( $A$ and $A^{\prime}$ ) was paired with a third description $(X)$ to form two message-pairs: $X+A$ and $X+A^{\prime}$. The $X$-passages 
that were selected had a choice pattern similar to that of $A$ and dissimilar to that of $A^{\prime}$; thus $X$ was relatively redundant with passage $A$ and nonredundant with passage $A^{\prime}$.

As noted above, redundancy was quantified (inversely) by means of the $D$-statistic. Profile comparisons in Experiment I yielded a mean $D$-score of .28 for the redundant message-pairs, with a range between .17 and .37 ; the nonredundant pairs had a mean $D$-score of .55 , with a range from .47 to .65 . Similar $D$-values were obtained for the redundant and nonredundant pairs in Experiment $\mathrm{II}^{1}$

\section{Presentation}

Messages were presented by means of an automatically controlled slide projector. In Experiment 1, the passages in each message-pair were exposed singly for $15 \mathrm{sec}$; in Experiment II, each message-pair was presented simultaneously for $30 \mathrm{sec}$. Following each pair of messages, the respondents attempted to identify the writers' intended referent, choosing from an array of 24 photographs. Subjects were given $1 \mathrm{~min}$ to respond and were permitted to make up to three (ordered) choices if they felt there was more than one "reasonable" possibility.

\section{Retention}

After reading the component passages of a given description-pair, the respondents were sometimes required to work on a series of rather difficult mental arithmetic problems before being presented with the array of referent-photographs. The arithmetic problems were presented in sets of 20 , and the respondents were given 2 min to complete as many problems in each set as they could, recording their answers in a response booklet.

Since Experiments I and II were ostensibly focussed on cognitive performance, the two types of problems (verbal and computational) were simply presented as tests of different cognitive abilities that were said to be equally important for the purpose of the research team.

Presentation of the arithmetic problems led to the creation of three delay conditions (retention intervals) in Experiment I: (a) no delay, (b) 2-min delay, and (c) 4-min delay. Experiment II involved just two delay intervals: (a) no delay and (b) 8-min delay. The delay conditions were presented without warning, so that when reading a given message-pair, the respondent did not know whether one or more sets of arithmetic problems would be interpolated before he was given an opportunity to make his decoding choice(s).

\section{Experimental Design}

Experiments I and II were both organized around a Latin square design, in which each respondent provided data for all of the experimental cells that resulted when the two levels of redundancy (high and low) were "crossed" with the various delay periods. The Latin squares were devised to produce overall performance measures that were unaffected by (a) differences in the codability of the various referent-photographs and (b) serial position effects. Table $I$ shows the basic design of Experiment I. The column headings represent the six main experimental conditions (two levels of redundancy $\times$ three levels of delay); the rows represent six different subgroups that were created to provide appropriate counterbalancing. Experiment II was based on a similar design scheme but involved only four main conditions (two levels of redundancy $\times$ two delay intervals); each respondent decoded eight different message-pairs (two representing each condition) and was given four scores that ranged between 0 and 2, to indicate the number of hits that he or she achieved in each condition.

1 The $D$-scores were calculated by comparing the proportion of norm group respondents who had selected the various response alternatives when decoding the component passages. 
TABLE 1

DESIGN FOR EXPERIMENT I

\begin{tabular}{|c|c|c|c|c|c|c|}
\hline \multirow{2}{*}{$\begin{array}{l}\text { Sub- } \\
\text { group }\end{array}$} & \multicolumn{3}{|c|}{ Low redundancy } & \multicolumn{3}{|c|}{ High redundancy } \\
\hline & Delay 0 & Delay 2 & Delay 4 & Delay 0 & Delay 2 & Delay 4 \\
\hline I & $\mathrm{F}^{a}$ & $\mathrm{C}$ & B & A & $\mathrm{D}$ & $\mathrm{E}$ \\
\hline II & A & D & $\mathrm{E}$ & $F$ & $C$ & B \\
\hline III & $\mathrm{C}$ & $\mathrm{E}$ & $F$ & D & B & A \\
\hline IV & D & B & A & $C$ & $\mathrm{E}$ & $\mathrm{F}$ \\
\hline V & B & $F$ & $\mathrm{C}$ & E & A & $\mathrm{D}$ \\
\hline VI & $\mathrm{E}$ & $A$ & $\mathrm{D}$ & B & $F$ & $\mathrm{C}$ \\
\hline
\end{tabular}

Note. The first message-pair of the experimental series was always based on photograph $\mathrm{A}$, the second on $\mathrm{B}$, the third on $\mathrm{C}$, etc. Thus, the experimental design involved an intentional confounding of picture-differences and differences associated with the ordinal position of the different message-pairs. Note that each combination of redundancy and delay (e.g., lowredundancy, no delay) appeared in a different ordinal position (1st. 2nd, 3rd, etc.) for each of the six experimental subgroups.

"Cell entries symbolize the correct referent-choices. In the actual experiment. the different photographs were identified by picture numbers, as in the original Frois-Wittmann series (Schlosberg, 1952).

\section{Subjects}

There were 60 respondents in Experiment $I$ and 56 in Experiment 1I. All respondents were enrolled at the University of Michigan and were paid for their efforts.

\section{Experiment I versus Experiment II}

The goals and methods of Fxperiments I and II were quite similar. Indeed. there were only two important procedural differences: (a) the method whereby the component passages were presented-successive (in Experiment I) versus simultaneous (in Experiment II); (b) the length of the retention interval, which was doubled from a maximum of $4 \mathrm{~min}$ in Experiment 1 to a maximum of $8 \mathrm{~min}$ in Experiment II.

\section{Results}

Figure 2 shows the results of Experiments $I$ and II. Figure 2 also provides information concerning the component descriptions, showing (a) the hit-rate for the more valid of the two passages in each message-pair, averaging the appropriate "input values" for the different referent-photographs, (b) the average hit-rate (across photographs) for the less valid passages, and (c) the mean of these two.

Overall, the data presented in Fig. 2 are consistent with the theorizing that motivated this research. Thus, in both experiments, the lowredundancy pairs yielded a higher hit-rate than the high-redundancy pairs, when the decoding choices were made without delay. While this effect was far from significant in Experiment $I$, where the com- 


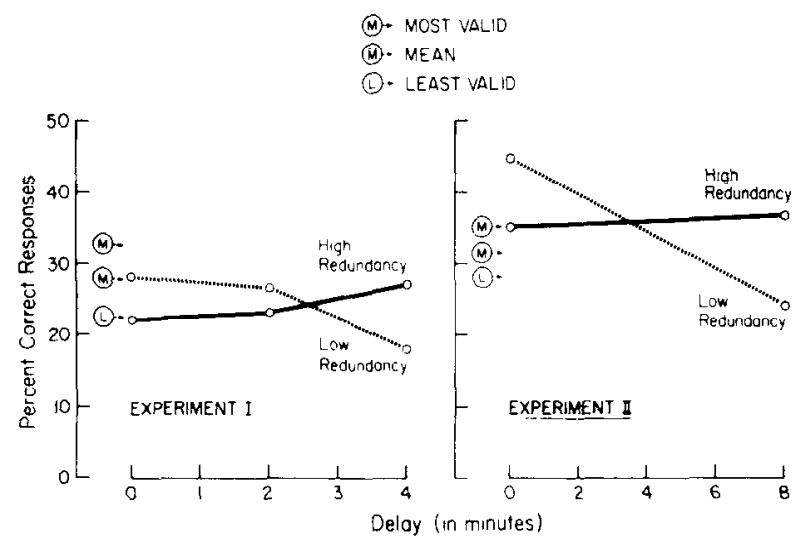

FIG. 2. Hit-rate results from Experiments I and II (first choices).

ponent passages were presented singly, the no-delay results proved more substantial in Experiment II, where the components were presented simultaneously $(p<.05$, one-tailed test). These data are consistent with the results reported by Manis and van Rooijen (1973).

The nonsignificant results that were obtained at the immediate response condition of Experiment I may be partly due to the scrial presentation technique that was used in this study; serial presentation probably involves more forgetting than simultaneous exposure, particularly for the passage that is presented first in a given pair. Retention failures of this sort would (theoretically) be especially troublesome when attempting to identify the referent for a nonredundant message-pair, which is of course the type of item that was initially expected to yield superior performance in the immediate response condition.

As shown in Fig. 2, following the interpolated arithmetic problems, the low-redundant pairs showed more deterioration than the high-redundant pairs. Indeed, the redundant pairs did not show any sign of deterioration (the modest "rise" that these pairs exhibit did not approach significance in either experiment). Despite the promising pattern of these results, which were in general accord with our theoretical expectations, the interaction between the retention interval and the redundancy variable was not significant in Experiment I, when only first choices were considered, but approached significance $(p<.065)^{2}$ when the respondents were given credit for a "hit" if they selected the correct photograph on any of their choices. In Experiment II, the interaction between redundancy and delay was unequivocal $(p<.025)$, presumably because the retention interval had been in- 
creased from a maximum of $4 \mathrm{~min}$ (in Experiment $\mathrm{I}$ ) to $8 \mathrm{~min}$ (in Experiment II).

The data from both experiments reflect a "crossover" pattern; Experiment II was particularly clear in showing that the redundant message-pairs yielded significantly better performance than the lowredundant pairs $(p<.05)$ following the interpolated arithmetic problems. This finding is inconsistent with a purely structural interpretation, for the structural theory suggests that with the passage of time, redundant and nonredundant message-pairs might yield comparable hit-rates, but it does not predict a crossover. The final (delayed) superiority of the redundant message-pairs thus seems more consistent with the differential retention theory; this theory was based on the assumption that the nonredundant pairs might be forgotten more rapidly than the redundant pairs, and hence it allowed for the possibility of a crossover. Unfortunately, these experiments did not include any verbal measures of message retention, which would have provided an additional check on the differential recall hypothesis.

\section{Component Hit-rates}

As shown in Fig. 2, Experiment I produced hit-rates that were generally lower than the mean of the contributing passages and were substantially poorer than the hit-rates of Experiment II. This was an unexpected outcome, and we believe that it is largely due to the fact that the subjects in this first study were quite tired, having just completed a demanding 1-hr experimental session in an uncomfortably hot room. In effect, this analysis suggests that for respondents like these, given their fatigue and possible boredom, the individual passages (presented singly) would probably have produced lower hit-rates than they did when presented to the original norm group. If this conjecture is correct, the relatively poor hit-rates that were elicited by the message-pairs in this study seem less anomalous. Note that in Experiment II, which used a sample of fresh (rested) respondents, the hitrates were generally better than the mean of the component passages, replicating the pattern that was originally observed in the study by Manis and van Rooijen (1973).

\section{EXPERIMENT III}

While Experiments I and II were concerned with the impact of redundancy when viewed from the perspective of the recipient (or "listener"), Experiment III focused on redundancy from the viewpoint of a sender who was attempting to transmit descriptive information to others. When considered in terms of uncertainty reduction, a rational sender might normally be expected to transmit nonredundant 
information, so as to maximize the diagnosticity of the information that was available to his receiver. On the other hand, previous research indicates that many receivers find it difficult to process nonredundant information; it seemed conceivable that the average speaker might have an implicit appreciation of this problem and might, as a consequence, transmit a disproportionate number of redundant messages.

\section{Method}

The respondents in this experiment were asked to play the role of "messagesenders." After decoding several sets of descriptive passages, the respondents were presented with a series of six items that took the following form: on each item the respondents were presented with a "given" description (the X-descriptions from Experiments I and II) and were shown the referent-photograph on which it was based. They were then told that since many of the passages were somewhat "vague," readers were sometimes unable to identify the correct target-photograph. Ostensibly to determine how well the respondents could supplement the information that was already available, each "given" passage was presented together with two "additional" descriptions ( $A$ and $A^{\prime}$ ) that were based on the same target-photograph and had yielded identical hit-rates when presented singly. The respondents' task was to select one of the descriptions from the "additional" pair such that this choice, when presented together with the "given" passage, would prove most effective in helping a naive reader (decoder) to select the proper target-referent. ${ }^{3}$ The six test items were presented in counterbalanced form, so that on three items the redundant passage (A) appeared as the first member of the additional pair and as the second member for the remaining three.

\section{Subjects}

The 80 respondents in this experiment were recruited from the student body at the University of Michigan and were paid for their participation.

\section{Results and Discussion}

The null hypothesis implied that by responding randomly, the average respondent would select the redundant alternative on half of the trials and the nonredundant passage on the remaining half. The obtained results show a modest but reliable departure from this pattern, for the mean respondent "overchose" the redundant descriptions, selecting them about $55 \%$ of the time $(p<.05)$. The results of Experiment III thus indicate that when presented with an opportunity to supplement a descriptive passage by choosing between two messages of equal quality (messages that produced identical hit-rates when pre-

${ }^{3}$ The test items were presented in two different formats. For some subjects, the various descriptions that comprised a particular item were shown together with the target-photograph. which was presented in isolation. For other subjects, the entire referent array was displayed on each item, with the target-referent being identified by number. The two presentation formats yielded virtually the same pattern of results. 
sented singly), our respondents were inclined to transmit redundant information in preference to material that was nonredundant.

This effect may partly derive from our respondents' reluctance to select messages that were at variance with the "given" description. That is, in their uncertainty as to the best of the available options, the respondents may have relied on the fact that the redundant passage (A) was rather similar to the information that was already "given" and hence appeared to have more consensual support than passage $\mathrm{A}^{\prime}$ as an appropriate characterization of the referent-photograph. This scenario would suggest that the preference for redundant material would be most pronounced if the "given" description appeared to emanate from a highly credible source rather than from one that was untrustworthy.

In contrast to the account that is sketched above, Experiment III may derive from a rather general (presumably learned) disposition to transmit relatively redundant messages. Although nonredundant message-pairs contain more information than redundant pairs (by definition), and sometimes yield better decoding performance, several lines of evidence suggest that people often find it difficult to process nonredundant messages, and this difficulty may underlie the results of Experiment III. First, it is important to note that the superiority of nonredundant messages seems restricted to those instances in which the listener (respondent) is free to make his or her decoding choice uithout delay. The interpolation of a relatively brief retention interval appears sufficient to reverse this pattern, however; hence nonredundancy constitutes a liability once memory demands become prominent.

Nonredundant information may also prove troublesome in situations where memory demands are minimal. For example, van Rooijen (1974) showed that respondents normally take longer to make their decoding choices when presented with nonredundant message-pairs, as compared with redundant pairs. Similarly, Kahneman and Tversky (1973) report that nonredundant information commonly leads to subjective uncertainty when people attempt to integrate psychometric information (test scores) in order to predict future behavior (college grades). The present results suggest that the respondents in Experiment III may have been implicitly sensitive to the difficulties presented by nonredundant information; acting accordingly, they were more likely to transmit redundant (rather than nonredundant) messages to their "listeners."

The results of Experiment III seem to complement previous research by Zajonc (1960) and Cohen (1961). These studies showed that when people are provided with information about a stranger with the expectation that they will subsequently be called upon to transmit 
this material to someone else, they retain a relatively consistent, unified (and presumably redundant) impression, compared to respondents who simply expect to serve as receivers of information. Cohen's study showed, moreover, that when the "transmitters" were asked if they wanted additional information beyond that which they had already acquired, they were likely to want narrow, one-sided material, in comparison to the "receivers," who preferred additional information that focused on both the good and the bad features of the individual who had been described to them.

The present studies highlight the complex role of redundancy in the communication process. Other things being equal, nonredundant information appears to facilitate communication (i.e., decoding performance) when the listener is free to process the information he has received without delay; redundant information appears to be more helpful, on the other hand, if the respondent is forced to store what he has learned in memory. Lastly, the advantages that redundancy provides may be particularly salient when we are called upon to transmit supplementary information to others, for the present results suggest that there may be a general "bias" that favors the transmission of relatively redundant messages.

\section{REFERENCES}

Cohen. A. A. Cognitive tuning as a factor affecting impression formation. Jommal of Personality, 1961, 29, 235-245.

Cronbach, L. J., \& Gleser, G. C. Assessing similarity between profiles, Psychological Bulletin, 1953, 50, 456-473.

Deese. J. The structure of associations in language and thought. Baltimore: Johns Hopkins Press. 1965.

Kahneman. D.. \& Tversky. A. On the psychology of prediction. Psychological Re'ie'w, 1973, 80, 237-251.

Manis, M., \& Armstrong, G. W. Contrast effects in verbal output. Journal of Experimental Social Psychology, 1971, 7, 381-388.

Manis, M.. \& van Rooijen, L. Integrating the information in referential messages. In R. L. Solso (Ed.), Contemporay issues in cognitive psychology: The Loysla symposium. New York: Winston \& Sons, 1973.

Osgood, C. E.. \& Suci. G. J. A measure of relation determined by both mean difference and profile information. Psychological Bulletin. 1952, 49, 251-262.

Schlosberg, H. The description of facial expressions in terms of two dimensions. Jowmal of Exporimental Psychology. 1952. 44, 229-237.

Trabasso. T.. \& Bower. G. H. Attention in learning: Theory and resectrch. New York: Wiley, 1968.

Underwood, B. J. The representativeness of rote verbal learning. In A. W. Melton (Ed.). Catcgories of human leaming. New York: Academic Press, 1964. Pp. $47-78$.

van Rooijen, L. B. Time processes in interpreting ambiguous descriptions of emotional states. Unpublished Ph.D. dissertation. University of Michigan. 1974.

Zajonc. R. B. The process of cognitive tuning in communication. Joumal of $A b$ normal and Social Psychology, 1960. 60, 159-167. 